\title{
Seroprevalence of Syphilis in Patient Attending Tertiary Care Hospital, Valsad, India
}

\author{
Hiral K. Patel*, Parimal H. Patel and Alka B. Nerurkar \\ Faculty Quarter, GMERS Medical College Campus, Halar road, Nanakwada, Valsad, India \\ *Corresponding author
}

\section{A B S T R A C T}

Syphilis is a sexually transmitted disease (STD) caused by Treponema pallidum. Prevalence of syphilis is changing in India recently because of availability of good laboratory diagnosis, treatment and increase health awareness. But it still remains as major public health problem with significant regional variations. Determine the seroprevalence of syphilis in various patients attending tertiary care hospital, Valsad. This retrospective study

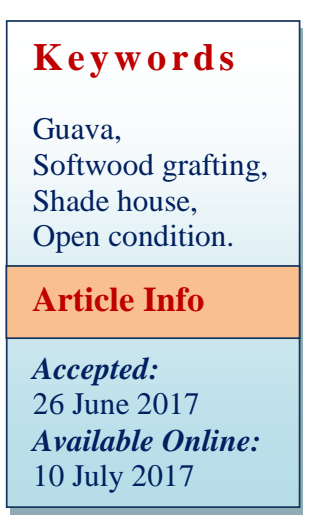
was conducted in a tertiary care teaching hospital Valsad in South Gujarat from May 2016 to April 2017 over a period of one year. Blood samples received from various patient groups including antenatal cases, patients attending sexually transmitted disease (STD) clinic and high risk cases were screened by Non specific treponemal test [Rapid Plasma Reagin (RPR) test] and specific treponemal test (Treponema pallidum Immunochromatography assay). From total 2938 samples only 63 were positive by qualitative RPR test. Of these 58 cases were confirmed by quantitative RPR test and Treponema pallidum immunochromatography assay. The overall seroprevalence found in this study was $1.97 \%$. The seroprevalence of patients attending the sexually transmitted infection clinic, antenatal group and among the HIV positive individuals were $4.11 \%$, $0.28 \%$ and $6.25 \%$ respectively. The present study found lower seroprevalence of syphilis in antenatal cases and in STD clinics. These findings could be interpreted as indicators of improved programmes for prevention and management of STDs. Even though the prevalence of syphilis is on the decline, screening of high risk populations should be continued to avoid the complications of undiagnosed and untreated syphilis.

\section{Introduction}

Syphilis, a sexually transmitted disease (STD) caused by Treponema pallidum, is associated with significant complications if left untreated and can facilitate the transmission and acquisition of HIV infection (World Health Organization, 2001). Maternal syphilis causes stillbirths and spontaneous abortion in $80 \%$ of cases and survivors are at risk of severe effects and long-term squealae. Screening for syphilis can prevent complications of syphilis and reduce transmission, which in turn will reduce the occurrence of congenital syphilis and transmission of human immunodeficiency virus (HIV) infection and other sexually transmitted infections (Schmid et al., 2007). It can be successfully controlled by effective public health measures due to the availability of a sound diagnostic test and effective economical treatment options. 
The World Health Organization estimates 5.6 million new syphilis cases in women and men aged 15-49 years globally. Based on data for 2005 to 2012, the estimated pooled prevalence for syphilis the global estimate was $0.5 \%$ and the regional values ranged from $0.2 \%$ to $1.8 \%$ (Newman et al., 2015). Various studies from India have shown varied rates of syphilis depending upon the population study. Studies from STD clinics and Antenatal groups have shown seroprevalence ranging from $5.4 \%$ to $8.2 \%$ and $0.84 \%$ to $0.98 \%$ respectively (Maity et al., 2011; Archana et al., 2014). Antibody detection by non treponemal/ cardiolipin and treponemal tests are the mainstay for diagnosing syphilis and for monitoring the success of subsequent antibiotic treatment (Centers for Disease Control and Prevention, 2010; Müller et al., 2014). So the main aim of the present study was to understand the occurrence of syphilis among various patient groups attending a tertiary care hospital.

\section{Materials and Methods}

This retrospective study was conducted in a tertiary care teaching hospital Valsad in South Gujarat from May 2016 to April 2017 over a period of one year. The samples were received from Patients at high risk for syphilis with clinical suspension of syphilis/as a part of routine screening for HIV seropositive patients attending ART center, Pregnant women attending antenatal clinics, STD clinic attendees without HIV and patients from OPDs or admitted to other wards of our tertiary care hospital. Serum samples from all patients were tested by qualitative Rapid Plasma Reagin RPR (nonspecific treponemal test) using standard procedures mentioned in the kit manual. Samples positive for qualitative RPR were subjected to a quantitative RPR test using serum dilutions from 1 in 2 up to 1 in 128. A specific treponemal test, Treponema pallidum immunochromatographic assays was performed on samples positive in the quantitative RPR tests. Samples positive in both tests were designated as seropositive for syphilis.

\section{Results and Discussion}

A total of 2938 samples were tested during this study period. Among them 63 were positive by qualitative RPR test. Of these 58 were positive by quantitative RPR and Treponema pallidum immune chromatographic assays. Overall seroprevalence of syphilis found in this study was $1.97 \%$.

Among the various sub-populations study, patients attending the sexually transmitted infection clinic showed a seroprevalence of $4.11 \%$. The antenatal group showed a seroprevalence of $0.28 \%$. Among the HIV positive individuals screened for syphilis, $6.25 \%$ were positive for treponemal antibodies. Miscellaneous groups of patients admitted to other broad specialties with features suggestive of secondary or tertiary syphilis were also screened. The seropositivity in this group was $2.76 \%$. Seroprevalence of syphilis among various sub-population groups is shown in table 1 .

Among these syphilis positive patients, 40(68.96\%) were male and 18 (31.04\%) were female. Majority of them were in the age group of $15-50 . i . e .(91.37 \%)$ The age and sex distribution of cases is shown in the table 2 .

A rise in the prevalence of syphilis has been observed in various studies in India and other countries (Centers for Disease Control and Prevention Sexually Transmitted Diseases Survillance, 2012; PHE, 2013; Ray et al., 2006). Seroprevalence of syphilis found in this study was $1.97 \%$, which is higher than the other hospital based study by Sethi et al., 
(2015) in which highest seroprevalence was $1.79 \%$ in year 2011. A rise in seroprevalence of syphilis was observed when all groups were analyzed together. Such findings have also been reported from USA, Germany, and Sweden. In USA, the rise in the prevalence has been ascribed to increased number of men who have sex with men (MSM) and reduction in safe sex practices among them. While in Europe, the rise attributed to increase number of MSM as well as increase testing in highrisk groups. A rising trend of secondary syphilis has been reported in hospital- based studies from India in past by Ray et al., (2006). This could be because of excessive reliance of preventive programs on the syndromic management of genital ulcer.

Only those patients positive by both quantitative RPR and Treponema pallidum immunochromatographic assay were analyzed to study the seroprevalence of syphilis. This was done to exclude the biological false positivity by RPR. In our study $0.17 \%$ biological false positivity by RPR was observed. Bala et al., (2013) reported 0.2\% biological false positivity by VDRL test and concluded that confirmatory tests should be performed in all sera reactive with VDRL regardless its titer.

The present study was determined the seroprevalence of syphilis in various subgroups of patients attending tertiary care hospital. Various studies from India have shown varied rates of syphilis depending upon the population study. Studies from STD clinics have shown seroprevalence ranging from $5.4 \%$ to $8.2 \%$. This study showed the lower Seroprevalence $(4.11 \%)$. These findings could be interpreted as indicators of improved programs for prevention and management of STDs as well as availability of treatment in STD clinics.

Table.1 Seroprevalence of syphilis among various sub-population groups

\begin{tabular}{|l|l|l|l|}
\hline Various groups & No.of tested & No. of positive & Percentage \\
\hline Antenatal cases & 1739 & 5 & $0.28 \%$ \\
\hline $\begin{array}{l}\text { Patients attending STD } \\
\text { clinics }\end{array}$ & 437 & 18 & $4.11 \%$ \\
\hline HIV positive & 400 & 25 & $6.25 \%$ \\
\hline Others & 362 & 10 & $2.76 \%$ \\
\hline
\end{tabular}

Table.2 Age and Sex distribution among Syphilis positive patients

\begin{tabular}{|l|l|l|l|}
\hline Age groups & Male & Female & Total \\
\hline$<15$ year & $1(1.73 \%)$ & $1(1.73 \%)$ & $2(3.46 \%)$ \\
\hline $16-50$ year & $36(62.05 \%)$ & $17(29.31 \%)$ & $53(91.36 \%)$ \\
\hline$>50$ year & $3(5.18 \%)$ & - & $3(5.18 \%)$ \\
\hline Total $(\mathrm{N}=58)$ & $40(68.96 \%)$ & $18(31.04 \%)$ & $58(100 \%)$ \\
\hline
\end{tabular}

Indian studies on syphilis from antenatal groups have shown a Seroprevalence of $0.84 \%$ to $0.98 \%$. Low seroprevalence of $0.28 \%$ was also seen in the current study. However, as syphilis can cause adverse outcomes of pregnancy in $80 \%$ of the cases, including stillbirths, abortions, perinatal death and neonatal infections in a significant number of cases, the importance of screening antenatal women for syphilis should always be highlighted (WHO, 2007). 
Co-infection rates of syphilis and HIV have been showing a worrisome trend in several countries, with urban outbreaks in men who have sex with men showing rates of $20 \%$ 73\% (Patton et al., 2014). In India, variable syphilis-HIV coinfection rates have been described (Shaw et al., 2011). Even though our study shows that $6.25 \%$ of diagnosed HIV patients were positive for syphilis, the results have to be interpreted with caution. The HIV positive individuals included in our study were symptomatic individuals who had presented to clinic for antiretroviral treatment. Therefore, all HIV positive individuals were not screened in the study unlike other studies which have screened large HIV positive cohorts. Syphilis and HIV co-infection presents a complex interaction. The increased incidence of HIV in homosexual and heterosexual individuals afflicted with STD, including syphilis is epidemiologically documented in numerous studies (Ross et al., 2014). HIV alters the course of syphilis as well as the response to treatment. Incidence of neurosyphilis in HIV infected individuals is high even when treated with recommended dosage (Tsai et al., 2012).

Patients from OPDs or admitted to other wards with features suggestive of secondary or tertiary syphilis were screened and seroprevalence of this group was 2.76\% which is similar with the study by Khan et al., (2014).

In the present study more males (68.96\%) were positive as compared to female (31.04\%), which correlates with the other study by Nishal et al., (2015). This might be due to higher social stigma of reporting to STI clinics in females. Majority of the patient were in the age group of 15-50 year as has been seen in other study as mainly because of high sexual activity in this age group (Nishal et al., 2015). The seroprevalence of syphilis found in this study was $1.97 \%$. We found lower seroprevalence in antenatal groups as well as in Patients attending STD clinics. Higher seroprevalence in HIV positive patients and patients from other wards and OPDS calls for continued and sustained effort for case detection, treatment and other preventive measure to contain the disease. Our study had several limitations. Its retrospective nature limited evaluation of several parameters like risk evaluation of particular groups such as men who have sex with men, rural and urban population etc. Secondly, it was a hospital based study and population-based community studies are likely to show different results.

\section{References}

Archana, B.R., Prasad, S.R., Beena, P.M., Okade, R., Sheela, S.R., Beeregowda, Y.C. 2014. Maternal and congenital syphilis in Karnataka, India. Southeast Asian J. Trop. Med. Public Health, 45(2): 430-43.

Bala, M., Singh, V., Muralidhar, S., Ramesh, V. 2013. Assessment of reactivity of three treponemal tests in non-treponemal nonreactive cases from sexually transmitted diseases clinic, antenatal clinic, integrated counselling and testing centre, other different outdoor patient departments/indoor patients of a tertiary care centre and peripheral health clinic attendees. Indian J. Med. Microbiol., 31(3): 275-27.

Centers for Disease Control and Prevention Sexually Transmitted Diseases Survillance. 2012. Atlanta: U.S. Department of health and human services.

Centers for Disease Control and Prevention. 2010. Sexually Transmitted Diseases Treatment Guidelines, 2010. MMWR Morb. Mortal Wkly. Rep., 59: 27-8.

Khan, S., G.A. Menezes, R. Dhodapkar, B.N. Harish. 2014. Seroprevalence of syphilis in patients attending a tertiary care hospital in Southern India. Asian Pac. J. Trop. Biomed., 4(12): 995-997. 
Maity, S., Bhunia, S.C., Biswas, S., Saha, M.K. 2011. Syphilis Seroprevalence among patients attending a sexually transmitted disease clinic in West Bengal, India. Jpn. J. Infect. Dis., 64(6): 506-508.

Müller, I., Brade, V., Hagedorn, H.J., Straube, E., Schörner, C., Frosch, M. 2014. Is serological testing a reliable tool in laboratory diagnosis of syphilis? Meta- analysis of eight external quality control surveys performed by the German infection serology proficiency testing program. J. Clin. Microbiol., 44: 1335-41.

Newman, L., Rowley, J., Vander Hoorn, S., Wijesooriya, N.S., Unemo, M., Low, N. 2015. Global Estimates of the Prevalence and Incidence of Four Curable Sexually Transmitted Infections in 2012 Based on Systematic Review and Global Reporting. PLoS ONE, 10(12): e0143304. doi:10.1371/journal.pone.0143304.

Nishal, P.K., Kapoor, A., Jain, V.K., Dayal, S., Aggrawal, K. 2015. Changing trends in acquired syphilis at tertiary care center of North India. Indian J. Sex Transm. Dis., 36(2): 149-153.

Patton, M.E., Su, J.R., Nelson, R., Weinstock, H. 2014. Centers for Disease Control and Prevention (CDC). Primary and secondary syphilis-United States, 20052013. MMWR Morb. Mortal Wkly. Rep., 63(18): 402-406.

PHE. 2013. Recent Epidemiology of Infectious syphilis and congenital syphilis Health protection report, 7.

Ray, K., Bala, M., Gupta, S.M., Khunger, N., Puri, P., Muralidhar, S. 2006 Changing trends in sexually transmitted infectionsat a Regional STD Centre in north India. Indian J. Med. Res., 124: 559-68.

Ross, M.W., Nyoni, J., Ahaneku, H.O., Mbwambo, J., McClelland, R.S., McCurdy, S.A. 2014. High HIV seroprevalence, rectal STIs and risky sexual behaviour in men who have sex with men in Dar es Salaam and Tanga, Tanzania. BMJ Open, 4(8): e006175.

Schmid, G.P., Stoner, B.P., Hawkes, S., Broutet, N. 2007. The need and plan for global elimination of congenital syphilis. Sex Transm. Dis., 34: S5-10.

Sethi, S., Mewara, A., Hallur, V., Prasad, A., Sharma, K., and Raj, A. 2015. Rising trends in syphilis in a tertiary care center in North India. Indian J. Sex Transm. Dis., 36(2): 140-143.

Shaw, S.Y., Deering, K.N., Reza-Paul, S., Isac, S., Ramesh, B.M., Washington, R. 2011. Prevalence of HIV and sexually transmitted infections among clients of female sex workers in Karnataka,India: a cross-sectional study. BMC Public Health, 11(Suppl 6): S4.

Tsai, H.C., Sy, C.L., Lee, S.S., Wann, S.R., Chen, Y.S. 2012. Optimal treatment for asymptomatic neurosyphilis. Int. J. STD AIDS, 23(10): 756-757.

WHO. 2007. The Global Elimnation of congenital Syphilis: Rationale and strategy for action, Geneva; World Health Organisation.

World Health Organization, Department of HIV/AIDS. 2001. Global prevalence and incidence of selected curable sexually transmitted diseases: overview and estimates. Geneva: World Health Organization.

\section{How to cite this article:}

Hiral K. Patel. 2017. Seroprevalence of Syphilis in patient attending Tertiary Care Hospital, Valsad. Int.J.Curr.Microbiol.App.Sci. 6(6): 2702-2706.

doi: https://doi.org/10.20546/ijcmas.2017.606.321 